\title{
Chapter 6 \\ Enhancing Safety Performance: \\ Non-technical Skills and a Modicum \\ of Chronic Unease
}

\author{
Rhona Flin
}

\begin{abstract}
Current debates on professionalism and safety cover a range of interpretative challenges and theoretical perspectives, as the workshop organized by FonCSI in 2015 revealed. One avenue for consideration was to address the question of the role of professionalism in the job with regard to safety. For example, should safety training just be part of normal job training or should it have a separate and distinctive position in the training curriculum? In this paper, I consider two ways in which safety training and safety thinking are being integrated into routine managerial and technical work. The first of these is behavioural, namely to focus on the non-technical skills (NTS) for a given job, as evidenced by the airlines' Crew Resource Management training and assessment programmes. This approach is now being adopted in other safety-critical sectors, such as acute medicine and offshore oil and gas operations. The second direction is more attitudinal in nature: it examines the relatively novel concept of chronic unease, derived from the High Reliability Organisation literature. These two approaches show that addressing both workplace behaviours (non-technical skills) and underlying attitudes to operational risks (chronic unease), can help to build protective skills for safety into the professional job repertoire.
\end{abstract}

Keywords Professionalism - Crew Resource Management - Chronic unease

\footnotetext{
R. Flin ( $\square)$

Robert Gordon University, Aberdeen, UK

e-mail: r.flin@rgu.ac.uk

(C) The Author(s) 2018

C. Bieder et al. (eds.), Beyond Safety Training, Safety Management,

https://doi.org/10.1007/978-3-319-65527-7_6
} 


\subsection{Introduction}

The opening position statement for the FonCSI workshop ${ }^{1}$ (see Introduction by Gilbert) came from a concern of the member companies, namely that training programmes in the field of industrial safety no longer appear to be yielding the expected results. This contribution is primarily directed at questions proposed for the FonCSI workshop on professionalism and safety

What part could professionalism in the job play in safety? Should safety training be incorporated into everyday practices and activities or should there be specific safety training? (Foncsi 2015)

The reason for choosing this topic is that much of my research has been founded, perhaps implicitly at times, on the assumption that enhancing job performance so that it is of better quality and efficiency will concomitantly enhance safety due to improved risk perception and risk management behaviours.

In this chapter, I first briefly discuss what I understand the term 'professionalism' to mean. It is not a topic I have ever studied and so I have attempted to set out my interpretation of how professionalism relates to workplace safety. I then discuss two areas where my own safety research has been located. These indicate two ways in which safety training and safety thinking can be integrated into routine work, at both operator and managerial levels. The first is behavioural, namely to focus on the non-technical skills for a given job, as evidenced by the airlines' Crew Resource Management training and assessment programmes. This approach is now being adopted in other safety-critical sectors, such as acute medicine and offshore oil and gas operations, typical for operational staff but in some cases also for managers. The second direction is more attitudinal in nature: it examines the relatively novel concept of chronic unease, derived from the High Reliability Organisation literature. This has been used at both operational and managerial levels. What is proposed is that addressing both non-technical skills, as well as underlying attitudes to operational risks (chronic unease), can help to build protective skills for safety into the professional job repertoire.

\subsection{What Is Professionalism?}

What does 'professionalism' in the job mean? Is it about having defined standards, specified programmes of education and qualification monitored by subject matter experts? The term 'profession' has a long history, traditionally referring to specialist occupations based on an extensive body of knowledge, such as law, divinity or medicine which have controlled qualifications and specified training leading to

\footnotetext{
${ }^{1}$ The two-day international workshop mentioned in the preface, organized by FonCSI in November 2015 and highlight of the project that led to this book (editors' note).
} 
membership of the professional body. Professionalism is a newer conceptualisation, reflecting an extended range of occupations now seen as professions and the idea of 'professional standards/professional behaviour' being seen as part of many modern jobs. Sociologists have engaged in extended debate about professionalism versus managerialism, normative versus ideological interpretations, the rise of professionalism and its implications for organisational life (Evetts 2003; Noordegraaf 2011). In relation to safety, we can distinguish between:

1. the increasing professionalization of the safety specialist, in the form of the safety adviser or safety manager and

2. embodying an additional focus on safety into the skills of the technical professional.

In relation to the first point, it is important to recognise that the safety specialists have a valuable role in many organisations, especially with regard to regulatory compliance, large scale audit, and design and implementation of safety management systems. My own work has been more concerned with the second approach. Namely, trying to identify how an appropriate skill set for enhancing safety can be identified so that this can be incorporated into professional development (whether technical or managerial).

\subsection{Crew Resource Management and Non-technical Skills}

One of the most obvious demonstrations of this approach of trying to build safety skills into general professional competence is Crew Resource Management (CRM). This is a training approach introduced by the aviation industry in the 1980s, following the realisation that a focus on technical skills was not sufficient. Accident analyses, which greatly benefitted from cockpit voice recorders, showed clearly that deficiencies in teamwork, leadership, decision making, situation awareness and communication were contributing to adverse events (Kanki et al. 2010). Of course, this was not to say that organisational factors, managerial behaviours, company culture and work conditions were not also exerting a powerful influence on airworthiness, technical reliability and flightdeck behaviours (Maurino et al. 1995). Notwithstanding these powerful influences on the behaviours of front-line staff, the personnel closest to the hazards may have the opportunity to enhance or diminish the level of flight safety by their actions. Their behaviour can increase exposure to risk (e.g. rule violation) or can be protective (e.g. by monitoring, and if necessary challenging, the actions of other crew members).

Task analyses using interviews, surveys, simulator observations and accident analyses were employed to identify pilots' CRM skills, which were essentially protective for safety, by reducing the incidence of error or by 'catching' or mitigating errors that occurred. Errors jeopardise efficiency, as well as safety. Improved communication enables smoother interaction between crew members and 
supporting personnel. Awareness of human performance limiting factors, such as stress and fatigue results in better self-monitoring and corrective action. Having determined the principal skill set, classroom and simulator-based training courses, called CRM training, were devised to teach pilots (and subsequently cabin crew and aviation engineers) the basic knowledge of the psychological principles underlying these non-technical skills and to show why the associated behaviours were protective for flight safety.

In European aviation, the regulator $\left(\mathrm{JAA}^{2}\right.$ at the time) when discussing Crew Resource Management introduced the term 'non-technical skills', essentially the cognitive and social skills that complemented the pilot's technical skills and enhanced efficiency and safety (Flin et al. 2008). The teaching and assessment of non-technical skills is obligatory for airline pilots in most countries. For example, in the UK, the Kegworth plane crash in 1989 (where British Midland pilots mistakenly shut off the working engine when the other was on fire) was such a strong demonstration that human error and teamwork failures were contributing to fatal accidents, that the Civil Aviation Authority (CAA) took the view that CRM had to be introduced, even though at the time there were only a few scientific studies on its effectiveness. In the ensuing years, there have been many advances in CRM training and in 2015 the European Aviation Safety Authority (EASA) released new guidance on Crew Resource Management (EASA 2015), an example of which is shown below.

CRM training should be conducted in the non-operational environment (classroom and computer-based) and in the operational environment (flight simulation training device (FSTD) and aircraft). Tools such as group discussions, team task analysis, team task simulation and feedback should be used.

CRM principles should be integrated into relevant parts of flight crew training and operations including checklists, briefings, abnormal and emergency procedures.

CRM training should address hazards and risks identified by the operator's management system described in ORO.GEN.200.

Whenever practicable, the compliance-based approach concerning CRM training may be substituted by a competency-based approach such as evidence-based training. In this context, CRM training should be characterised by a performance orientation, with emphasis on standards of performance and their measurement, and the development of training to the specified performance standards.

One of the strengths of the CRM approach is that the training content is based on underlying scientific evidence from psychology, physiology or other relevant disciplines (Kanki et al. 2010). Thus it strives to continually develop and foster evidence-based practice. Another fundamental principle of CRM is that the training content should be designed to address current operational issues and to reflect learning from adverse events and near misses. These are both illustrated in the section below.

\footnotetext{
2Joint Aviation Authorities (editors' note).
} 


\subsubsection{Startle Effects}

One example of this process of incorporating learning from adverse events into CRM training relates to the Air France accident (2009). An Airbus (AF447) crashed into the Atlantic Ocean when flying between Rio de Janeiro and Paris (BEA 2012). One of the contributing factors to the accident was that the pilots had apparently become startled by rapid changes in aircraft state. The EASA (2015, p. 5) guidance, mentioned above, addresses this issue.

(3) Resilience development

CRM training should address the main aspects of resilience development. The training should cover:

(i) Mental flexibility

Flight crew should be trained to:

(A) understand that mental flexibility is necessary to recognise critical changes;

(B) reflect on their judgement and adjust it to the unique situation;

(C) avoid fixed prejudices and over-reliance on standard solutions; and

(D) remain open to changing assumptions and perceptions.

(ii) Performance adaptation Flight crew should be trained to:

(A) mitigate frozen behaviours, overreactions and inappropriate hesitation; and

(B) adjust actions to current conditions.

(4) Surprise and startle effect

CRM training should address unexpected, unusual and stressful situations. The training should cover:

(i) surprises and startle effects; and

(ii) management of abnormal and emergency situations, including:

(A) the development and maintenance of the capacity to manage crew resources;

(B) the acquisition and maintenance of adequate automatic behavioural responses; and

(C) recognising the loss and re-building situation awareness and control." (p. 5)

These additions to CRM training had to be implemented by European operators by October 2016. This is an excellent example of how fundamental training for a professional group is continually reviewed and revised (in this case by an international regulatory body) to take into account emerging issues relating to safety that have not previously been recognised to this degree.

As mentioned above, research evidence is used to develop the content of CRM training and this has also been true for the startle effect phenomenon. When EASA were reviewing their European CRM guidance, specific research findings on startle effects were sought and considered. In the USA, the Federal Aviation Administration (FAA) commissioned research into this phenomenon (e.g. Rivera et al. 2014) and on the more general effects of acute stress on aircrew performance (Dismukes et al. 2015). There are only a limited number of studies but these have shown individual variation in response and recovery patterns, as well as pilots' 
awareness of this effect (Martin et al. 2015, 2016). And of course, startle effects are not peculiar to pilots. Recent studies in healthcare indicate similar patterns of reaction to unexpected events with concomitant delays in decision making and responsive action during resuscitation ( $\mathrm{Lu}$ et al. 2015).

\subsubsection{CRM Beyond the Flightdeck}

The CRM/non-technical skills approach has now extended into many other occupations, including the mariners and ship engineers; railway workers, miners, systems analysts (Flin et al. 2008, 2014). In healthcare, there has been particular interest from members of operating theatre teams with non-technical skill sets developed for anaesthetists (ANTS); scrub nurses (SPLINTS); anaesthetic practitioners (ANTS-AP) and surgeons (NOTSS). There are now training courses on non-technical skills provided for these occupations and the Royal Australasian College of Surgeons incorporated the NOTSS framework into their new professional standards (Flin et al. 2015). As suggested above, this shows how an increased emphasis on behaviours targeted to improve safety (via a non-technical skills approach) can be adopted as part of existing professional development.

The main objective of this CRM/ NTS training and assessment has always been to improve safety/reduce accidents, hence the behavioural rating scales tools to measure performance on non-technical skills are phrased in the language of safety. For example, in the NOTECHS system for pilots (van Avaermaete and Kruijsen 1998; O'Connor et al. 2002), the scale descriptors use explanatory terms such as

'behaviour directly endangered flight safety' or 'behaviour enhances flight safety'

for very poor and good performance respectively. But the behavioural examples (markers) in such systems relate not to specific safety-related activities but to normal task operations. Thus the underlying premise is that better demonstration of skills such as leadership, teamwork, decision making during task execution will benefit safety. The medical professionals who developed non-technical skills frameworks and associated behavioural rating systems (e.g. ANTS, NOTSS) have adopted the same type of scale descriptors (Flin et al. 2015), in this case with the purpose of emphasising that patient safety is of paramount importance.

Following the blowout on the Deepwater Horizon drilling rig (2010) in the Gulf of Mexico that killed 11 workers and injured a further 50, as well as creating an enormous marine pollution event (Report to the President 2011), the offshore oil and gas industry became interested in applying CRM to enhance safety (Flin et al. 2014). Social scientists' analyses of the accident show clearly how failures in non-technical skills could have contributed to the trajectory of this event (Hopkins 2012; Reader and O'Connor 2014; Roberts et al. 2015b). In order to design customised training for drillers and other well control specialists, detailed task analyses are required to pinpoint the most important non-technical skills that can help to 
protect the safety of the well (Roberts et al. 2015a). In a number of drilling companies, these skills are being taught alongside the technical skills of well control, especially where there are simulation facilities available that allow for demonstration and feedback.

The evaluation literature on CRM and safety outcomes is somewhat limited in aviation (low accident rates offer insufficient outcome data) although there have been meta-analyses (e.g. O'Connor et al. 2008). The more recent introduction of non-technical skills/CRM to the world of healthcare means that as a technique it is being scrutinised by a new level of rigour, given medical professionals' concern with treatment efficacy and willingness to measure error rates and outcomes. Thus there is an emerging database of studies examining the relationships between technical skills, non-technical skills, error and safety or other performance metrics. These generally indicate positive, if patchy, relationships (Hull et al. 2012) but there is an emerging message that focussing on improving the non-technical skills required for both routine and abnormal task activities can improve safety.

In the following section, I consider a second approach that is being adopted by some companies which is not just to consider behaviours but also to foster particular attitudes or 'mind sets' that will drive the choice of behaviours that should enhance safety. This is another approach to building safety professionalism into everyday task activities, and the mental state in question is called 'chronic unease'.

\subsection{Chronic Unease}

In a work environment that has few accidents, even though there are significant hazards present, there is a likelihood that the risks are underestimated leading to a false sense of comfort or complacency. A report by Cass Business School (2011), investigated major corporate crises (explosions, fires, product-related and supply chain crises, and $\mathrm{IT}^{3}$ problems) and identified failures at board level in these organisations. There was an inability to recognise potential risks and engage with them, a tendency to ask fewer questions when things were going well and not recognising changes in the corporate environment.

Recent industrial interest in applying the concept of 'chronic unease' to managerial and operational thinking on safety matters is an attempt to address this type of problem. The concept comes from the literature on 'high reliability organisations' (HRO). A key HRO characteristic is the lack of complacency about risks. For instance, with regard to the structural failures that caused the Alexander Keilland drilling rig accident in Norway, Weick (1987, p. 119) commented

Part of the mind-set for reliability requires a chronic suspicion that small deviations may enlarge, a sensitivity that may encourage a more dynamic view of reliability.

\footnotetext{
${ }^{3}$ Information Technology (editors' note).
} 
The term 'chronic unease' was introduced by Reason (1997) to capture tendencies of wariness towards risks, thus as a contrast to complacency. He described it as resulting from an absence of negative events, leading 'people [to]forget to be afraid' (p. 39). The Oxford English dictionary defines 'unease' as a form of discomfort and distress, related to strain and representing a feeling of concern.

In a similar vein, the HRO literature discusses alertness and good management of risks under the label of 'mindfulness' in organisations. Weick and Sutcliffe (2006) describe mindful organisations as:

1. dealing with risks by investing substantial resources, both financial and attentional,

2. early detection of issues,

3. pre-occupation with failure,

4. reluctance to simplify,

5. sensitivity to nuances that can lead to failure,

6. commitment to resilience and

7. willingness to defer to experts.

The resilience literature uses the term 'restless mind' to label awareness that things can go wrong and alertness to weak signals (Westrum 2008). Likewise, Pidgeon (2012) writes of 'safety imagination' to describe inadequate appreciation of risk in relation to the Fukushima nuclear power plant accident in Japan in 2011.

My research group had been studying managers' safety leadership and safety commitment (Agnew and Flin 2014; Flin 2006; Fruhen et al. 2014) and we were sponsored by a multinational oil and gas company to work on a study of chronic unease in managers. This company had already developed safety materials for operational staff which emphasised the importance of having a sense of respectful unease for ever-present risks in the work environment and confirmed the importance of vigilance and attention to weak signals. The focus of our project was on how more senior managers might experience chronic unease, how that could influence their behaviour and whether they felt this was beneficial for safety and for the business more generally.

Despite the prevalence of the term 'chronic unease' in the high reliability organisation (HRO) literature, there was limited evidence to enable a definition or operationalisation of this concept. To develop a better understanding of chronic unease, we conducted a literature search using this term (Fruhen et al. 2014). We only found descriptions of chronic unease in 9 articles. These were coded resulting in the identification of five themes: pessimism, propensity to worry, vigilance, requisite imagination and flexible thinking. From the descriptions in the literature and with reference to related conceptualisations, we proposed the following components:

- Pessimism is a disposition that drives individuals to anticipate failures and expect negative events and therefore may promote chronic unease about safety. Pessimism is not concerned with emotions and somatic reactions, but rather represents an attitude towards the future. 
- Propensity to worry is a tendency towards experiencing an emotional reaction with regard to possible failure. This could be characterised as a personality trait (worry regularly about many things) or as a more transient state (to worry about a particular event). It is a subcomponent of anxiety, described as specific to cognition that is characterised by concern about future events.

- Vigilance is usually defined in terms of an observer's ability to maintain attentional focus and to remain alert to stimuli over prolonged periods of time. Thus it concerns the detection of signals in the environment.

- Requisite imagination was defined by Adamski and Westrum (2003, p. 195)

the fine art of anticipating what might go wrong.

It is not primarily concerned with the exploration of current states of problems, but with the ability to project their future development.

- Flexible thinking relates to creative problem solving which typically involves divergent thinking. We suggested that chronic unease promotes this mode of cognition.

We proposed a preliminary conceptualisation of chronic unease based on these attributes, arguing that this particular mental state may be desirable for managers in relation to the control of risks. Desirable because the feelings of unease may lead them to be more attentive to risk information and to incorporate a safety dimension into their operational decision making.

In a second study, we carried out semi-structured interviews with 27 senior managers from several companies in the energy sector (Fruhen and Flin 2016). The aim of the study was to determine if the five components identified in the literature review would be evident in the managers' responses when discussing their safety leadership practices. We were also interested in how a sense of chronic unease would affect the managers' behaviours. Content analysis of the interview transcripts identified flexible thinking most frequently, followed by pessimism, propensity to worry, vigilance and requisite imagination. Flexible thinking was frequently also coded as a behaviour, suggesting it to be a partially observable response to chronic unease. Other behaviours that emerged as related to chronic unease were demonstrating safety commitment, transformational and transactional leadership styles, and seeking information. Chronic unease was described as having positive effects on safety, positive and negative effects on team interaction and negative effects on business and the managers' personal outcomes. We concluded that the five components provide a basis for the measurement of chronic unease and suggested behaviours and responses that should be considered in its future investigation. Figure 6.1 provides a proposed model of how the components and associated behaviours might be related.

In terms of increasing the professionalization of safety in managers, it is suggested that attention should be paid to these underlying characteristics and attitudinal states ('mind-set for reliability'), as well as considering the related behaviours. The extent to whether 'chronic unease' in managers can be developed is still to be 


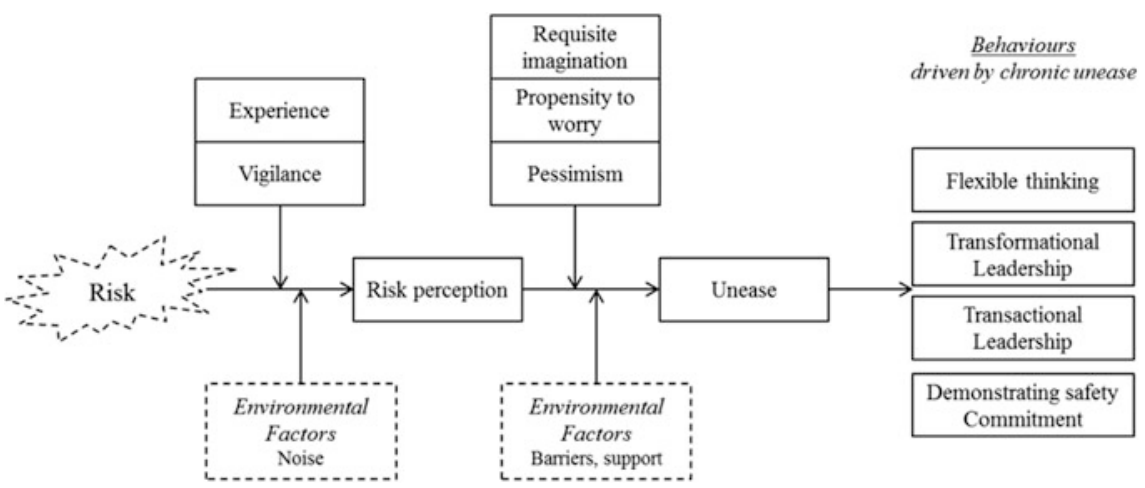

Fig. 6.1 Proposed model of the influence of chronic unease on managers' behaviours (Fruhen and Flin 2016). Reprinted from the Journal of Risk Research with the permission of Taylor and Francis

determined. Managerial safety training which endeavours to increase risk awareness, to demonstrate the personal consequences for managers of having a major accident and to show how companies can become complacent is essentially trying to increase a sense of unease.

When introducing the topic of chronic unease, I am often asked- 'So how much chronic unease is desirable?' Certainly a high level of chronic unease for safety could have negative consequences for individuals who are consumed by worry and constantly fearful of catastrophic events on their worksites. Of course, any extreme mental state can be harmful; at an abnormal level there could be reduced well-being, stress and even clinical anxiety and depression. Thus we proposed that the relationship between chronic unease and efficacy in safety management probably has a curvilinear nature (Flin and Fruhen 2015). Too little unease and the resulting complacency could mean that warning signals are ignored, ambiguities are marginalized, there is no systematic search for negative indicators, and adverse consequences are rarely considered. Too much unease and the manager could be disabled by anxiety with consequent deleterious effects on decision making, action and mental health. At the optimal level, which will be individually determined, the sense of chronic unease about organisational safety prompts a continued search for hidden threats, the extended consideration of ambiguities and anomalies, and the appreciation of disconfirming evidence.

This suggestion of a curvilinear relationship between for chronic unease and managerial performance is based on Janis and Mann's (1977) conflict model of stress and decision making, where they described coping patterns in decision conflict situations (i.e. ambiguity about the best option) with distinctive levels of stress. They describe various states including 'hypervigilance' where there is recognition of the serious risks in the alternative courses of action. In this case the stress level is extreme (cf. high chronic unease) creating a state akin to panic with the individual preoccupied with the threatened losses. Resulting behaviours can include impulsive actions, vacillation and simplistic, repetitive thinking. We argued 
(Flin and Fruhen 2015) that such high levels of anxiety might exist because a manager is not well-suited to the job, or has insufficient knowledge and support. Alternatively, such high anxiety could be a strong indication that there are uncontrolled risks and that continuing with workplace operations could result in an accident.

It was suggested that the desirable level of chronic unease for safety is in the mid-range, perhaps this could be called 'a modicum of unease'. This is similar to what Janis and Mann called vigilance which was associated with a moderate level of stress. In this case, they described the decision maker recognizing that there are serious risks imbued in competing alternatives, but having confidence about the likelihood of finding an adequate solution in the available time. Clearly there would be marked individual differences in what level of unease would produce discomfort in managers and the types of risks that would most concern them. The professional implications of chronic unease may be more pertinent to organisational selection practices than to safety training.

\subsection{Conclusion}

As Gilbert (see Introduction) points out in his opposition statement regarding the ineffectiveness of safety training, there is a need to make a finer grained assessment of exactly where there appears to be no or minimal return on investment. It is likely that many safety training courses such as on hazard awareness, use of protective techniques, event analysis, risk protection measures, do deliver the anticipated developments in knowledge, skills and attitudes. Safety training courses in CRM and non-technical skills can also broaden the range of risk awareness, especially to show how social or intrapersonal factors can increase the risk level during task execution. These courses can also focus on specific methods for altering behaviours, for example, relating to speaking up, listening, conducting handovers, problem solving, task briefing which can have longer term effects on hazard awareness levels, as well as shifting norms of acceptable behaviour to improve the safety culture. However, the evidence on effectiveness for both technical and non-technical safety training can be limited and one component of increasing the professionalism of safety may be a requirement for organisations to spend more time and money evaluating the longer term impact of their safety training and other safety interventions. This would require baseline data to be gathered on knowledge, skills and attitudes prior to the training programme and more use of randomised control designs to enable a robust assessment of treatment effects. To enhance the professionalism of safety, whether by developing safety professionals or increasing safety awareness and related skills of operational staff, a strong evidence base is required to determine what should be trained and how the skills can be developed and maintained. In addition, more attention may need to be devoted to personal attributes, such as having a sense of chronic unease when dealing with hazardous activities. In summary, addressing both workplace, on-task behaviours 
(non-technical skills), as well as underlying attitudes to operational risks (chronic unease), can help to build protective skills for safety into the professional job repertoire.

\section{References}

Adamski, A. \& Westrum, R. (2003). Requisite imagination: The fine art of anticipating what might go wrong. In E. Hollnagel (Ed.), Handbook of Cognitive Task Design (pp. 193-220). Mahwah, NJ: Lawrence Erlbaum.

Agnew, C. \& Flin, R. (2014). Senior charge nurses' leadership behaviours in relation to hospital ward safety: A mixed method study. International Journal of Nursing Studies, 51, 768-780.

BEA. (2012). Final Report. Flight AF 447 on $1^{\text {st }}$ June 2009 A330-203. Paris: Bureau d'enquêtes et d'analyses pour la sécurité de l'aviation civile.

Cass Business School. (2011). Roads to Ruin: A Study of Major Risk Events: Their Origins, Impact and Implications. London: Airmic.

Dismukes, K., Goldsmith, T. \& Kochan, J. (2015). Effects of acute stress on aircrew performance: Literature review and analysis of operational aspects. NASA Report: NASA/TM-2015218930.

EASA. (2015). AMC1 ORO.FC.115 Crew resource management (CRM) training. Annex II to Decision 2015/022/R. Cologne: author.

Evetts, J. (2003). The sociological analysis of professionalism. International Sociology, 18, 295415.

Flin, R. (2006). Erosion of managerial resilience: Vasa to NASA. In E. Hollnagel, D. Woods \& N. Leveson (Eds.), Resilience Engineering. Aldershot: Ashgate.

Flin, R. \& Fruhen, L. (2015). Managing safety: Ambiguous information and chronic unease. Journal of Contingencies and Crisis Management, 23, 84-89.

Flin, R., O'Connor, P. \& Crichton, M. (2008). Safety at the Sharp End. A Guide to Non-Technical Skills. Aldershot: Ashgate.

Flin, R. Wilkinson, J. \& Agnew, C. (2014). Wells Operations Crew Resource Management. International Oil and Gas Producers Report 501. London: IOGP.

Flin, R., Youngson, G. \& Yule, S. (2015). Enhancing Surgical Performance. A Primer on Non-Technical Skills. London: CRC Press.

Foncsi. (2015). Is professionalization a safety issue... or the other way around? Strategic analysis call for papers. Foundation for an industrial safety culture. https://www.foncsi.org/en/research/ research-themes/professionalisation-folder.

Fruhen, L., Flin, R. \& McLeod, R. (2014). Chronic unease for safety in managers: a conceptualisation. Journal of Risk Research, 17(8), 969-979.

Fruhen, L., Mearns, K., Flin, R. \& Kirwan, B. (2014). Skills, knowledge and senior managers' demonstrations of safety commitment. Safety Science, 69, 29-36.

Fruhen, L. \& Flin, R. (2016). 'Chronic unease' for safety in senior managers: an interview study of its components, behaviours and consequences. Journal of Risk Research, 19, 645-663.

Hopkins, A. (2012). Disastrous Decisions. Sydney: CCH Australia.

Hull, L., Arora, S., Aggarwal, R., Darzi, A., Vincent, C., \& Sevdalis, N. (2012). The impact of nontechnical skills on technical performance in surgery: a systematic review. Journal of the American College of Surgeons, 214, 214-230.

Janis, I. \& Mann, L. (1977). Decision Making: A Psychological Analysis of Conflict, Choice and Commitment. New York: Free Press.

Kanki, B., Helmreich, R. \& Anca, J. (2010) (Eds.). Crew Resource Management ( $2^{\text {nd }}$ edition). San Diego: Academic Press. 
Lu, S., Waller, M., Kaplan, S., Watson, A., Jones, M. \& Wessel, D. (2015). Cardiac resuscitation events: One eyewitness is not enough. Pediatric Critical Care Medicine, 16, 335-342.

Martin, W., Murray, S., Bates, P. \& Lee, P. (2015). Fear-potentiated startle: A review from an aviation perspective. International Journal of Aviation Psychology, 25, 97-107.

Martin, W., Murray, P., Bates, P. \& Lee, P. (2016). A flight simulator study of the impairment effects of startle on pilots during unexpected critical events. Aviation Psychology and Applied Human Factors, 6, 24-32.

Maurino, D., Reason, J., Johnston, N. \& Lee, R. (1995) Beyond Aviation Human Factors. Aldershot: Ashgate.

Noordegraaf, M. (2011) Risky business. How professionals and professional fields (must) deal with organizational issues. Organization Studies, 32, 1349-1371.

O'Connor, P., Hörmann, H. J., Flin, R., Lodge, M., Goeters, K. M., \& JARTEL Group, T. (2002). Developing a method for evaluating Crew Resource Management skills: A European perspective. The International Journal of Aviation Psychology, 12, 263-285.

O'Connor, P., Campbell, J., Newon, J., Melton, J. Salas, T \& Wilson, K. (2008). Developing a method for evaluating Crew Resource Management skills: a European perspective. International Journal of Aviation Psychology 18, 353-368.

Pidgeon, N. (2012). Complex organizational failures: Culture, high reliability, and lessons from Fukushima. The Bridge, 42, 17-22.

Report to the President. (2011). National Commission on the Deepwater Horizon Oil Spill and Offshore Drilling. Washington, DC: US Government Printing.

Reader, T., \& O'Connor, P. (2014). The Deepwater Horizon explosion: nontechnical skills, safety culture, and system complexity. Journal of Risk Research, 17, 405-424.

Reason, J. (1997). Managing the Risks of Organizational Accidents. Aldershot: Ashgate.

Rivera, J., Talone, A., Boesser, C., Jentsch, F. \& Yeh, M. (2014). Startle and surprise on the flight deck: Similarities, differences and prevalence. In Proceedings of the Human Factors and Ergonomics Society Annual Meeting (Vol. 58, No. 1, pp. 1047-1051). Los Angeles, CA: SAGE Publications.

Roberts, R.C., Flin, R., \& Cleland, J. (2015a). Staying in the zone: Offshore drillers' situation awareness. Human Factors, 57, 573-590.

Roberts, R., Flin, R. \& Cleland, J. (2015b). 'Everything was fine' An analysis of the drill crew's situation awareness on Deepwater Horizon. Journal of Loss Prevention in the Process Industries, 38, 87-100.

Weick, K. (1987). Organizational culture as a source of high reliability. California Management Review, 24, 112-127.

Weick, K. \& Sutcliffe, K. (2006). Mindfulness and the quality of organizational attention. Organizational Science, 17, 514-524.

Westrum, R. (2008). Resilience and restlessness. In E. Hollnagel, C. Nemeth \& S. Dekker (Eds.). Remaining Sensitive to the Possibility of Failure. 1-2. Aldershot: Ashgate.

van Avermaete, J. \& Kruijsen, E. (1998) (Eds.). NOTECHS. The Evaluation of Non-Technical Skills of Multi-Pilot Aircrew in Relation to the JAR-FCL Requirements. Final Report NLR-CR-98443. Amsterdam: National Aerospace Laboratory (NLR). 
Open Access This chapter is licensed under the terms of the Creative Commons Attribution 4.0 International License (http://creativecommons.org/licenses/by/4.0/), which permits use, sharing, adaptation, distribution and reproduction in any medium or format, as long as you give appropriate credit to the original author(s) and the source, provide a link to the Creative Commons license and indicate if changes were made.

The images or other third party material in this chapter are included in the chapter's Creative Commons license, unless indicated otherwise in a credit line to the material. If material is not included in the chapter's Creative Commons license and your intended use is not permitted by statutory regulation or exceeds the permitted use, you will need to obtain permission directly from the copyright holder.

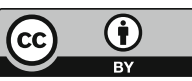

